\title{
The Rescue and the Selection of the Thermally Stabled Type 0 Vaccine Candidate Strains of Foot- and-mouth Disease Virus
}

Dong Li ( $\sim$ lidong@caas.cn )

Lanzhou Veterinary Research Institute https://orcid.org/0000-0002-9101-0241

\section{Ya Gao}

Lanzhou Veterinary Research Institute

Pinghua Li

Lanzhou Veterinary Research Institute

\section{Xueqing Ma}

Lanzhou Veterinary Research Institute

\section{Xingwen Bai}

Lanzhou Veterinary Research Institute

\section{Pu Sun}

Lanzhou Veterinary Research Institute

\section{Ping Du}

Lanzhou Veterinary Research Institute

Hong Yuan

Lanzhou Veterinary Research Institute

\section{Yimei Cao}

Lanzhou Veterinary Research Institute

Kun Li

Lanzhou Veterinary Research Institute

\section{Yuanfang Fu}

Lanzhou Veterinary Research Institute

Jing Zhang

Lanzhou Veterinary Research Institute

\section{Huifang Bao}

Lanzhou Veterinary Research Institute

\section{Yingli Chen}

Lanzhou Veterinary Research Institute

\section{Zhiyong Li}

Lanzhou Veterinary Research Institute

\section{Zengjun Lu}


Lanzhou Veterinary Research Institute

\section{Zaixin Liu}

Lanzhou Veterinary Research Institute

\section{Research Article}

Keywords: Foot-and-mouth disease virus (FMDV), Thermostability, Rescue, Selection, Potential vaccine strain

Posted Date: February 17th, 2021

DOl: https://doi.org/10.21203/rs.3.rs-207421/v1

License: (9) This work is licensed under a Creative Commons Attribution 4.0 International License. Read Full License 


\section{Abstract}

The inactivated vaccines of Foot-and-mouth disease virus (FMDV) have been used widely in the world to control Foot-and-mouth disease (FMD). But the virions (146S) of this virus are easily dissociated into pentamer subunits (12S), thus limits the immune protective efficacy of the inactivated vaccines when the temperature is higher than $30^{\circ} \mathrm{C}$. The cold-chain system can maintain the quality of the vaccines, but that is usually not reliable in limited resource settings. Thus, it is imperative to improve the thermostability of vaccine strains to guarantee the vaccines' quality. In this study, 4 recombinant FMDV strains containing single or multiple amino acids substitutions in the structural proteins (SP) were rescued by using a preconstructed FMDV type 0 full-length infectious clone (pO/DY-VP1). The assays used here indicated that the single or multiple amino acids substitutions in SP may affect the viral replications to different degrees. Furthermore, the heat and acid stabilities of the recombinant viruses were significantly improved comparing with the parental virus. Three well thermally stable strains of recombinant viruses (rHN/DYVP1 ${ }^{\text {Y2098F }}$, rHN/DY-VP1 V2090A-S2093H and rHN/DY-VP1 V2090A-S2093H-Y2098F) were selected for inactivated vaccine to immunize pigs. Blood samples were collected every week to prepare sera. Meanwhile the effects of mutations in SP amino acids on the antigenicity were analyzed by viral neutralization test, which showed that the substitutions of S2093H, Y2098F and S2093H-Y2098F did not affect the immunogenicity. In addition, comparing to the parental virus, Y2098F mutation could increase the thermostability significantly $(\mathrm{p}<0.05)$. In conclusion, the $\mathrm{rHN} / \mathrm{DY}-\mathrm{VP} 1^{\mathrm{Y} 2098 \mathrm{~F}}$ mutant is considered as a potential vaccine strain in the future.

\section{Introduction}

Foot-and-mouth Disease virus (FMDV) with seven serotypes (include O, A, Asia 1, C, SAT1-3) is the pathogen of Foot-and-mouth Disease (FMD), an acute and highly contagious infectious disease that affects cloven-hoofed animals such as pigs, cattle and sheep [1,2]. Due to its serious impact on the economy, politics and society of endemic countries or regions, FMD has been listed as the top 15 class A severe animal infectious diseases by the Office International des Epizooties (OIE) [3]. Nowadays, FMD control is predominantly carried out by the vaccination, of which the chemically inactivated vaccine is still widely applied to prevent and control FMD. The studies have shown that FMDV capsids are generally unstable, especially of the viral strains of the types 0 and SAT serotypes, which are very sensitive to temperature and $\mathrm{pH}$ [4]. When the temperature is higher than $30^{\circ} \mathrm{C}$ or the $\mathrm{pH}$ is less than 7 , the capsid of FMDV will depolymerize [4,5], leading to the decrease of the effective antigen (146S) content in vaccine so as to affect their immune protection efficacies [6-8]. At present, the main concern relevant to the conventional FDM vaccines are the antigen instability[9], the short-lived immunity with approximately 6 month duration and the inability to stimulate the high cell-mediated immunity with the animals [10-12].

To maintain the efficacy of the FMD vaccine, the expensive cold chain transportation system is required $[13,14]$. However, cold chain storage and transportation are not completely reliable. For example, there is lack of the extensive and the reliable frozen facilities in some terminal nodes of vaccine transportation. So, it's difficult to guarantee the efficacy of vaccines. Statistics show that nearly $50 \%$ of vaccines are 
discarded and wasted every year in the world, due to poor storage management during transportation [15]. In addition, it is also expensive to keep the vaccine in a relatively stable cold environment for a long period. According to statistics, the expenditure on vaccine cold-chain system in some developing countries or regions is up to $80 \%$ of the total financial cost $[16,17]$. Therefore, improving the thermostability of the vaccine virus strain and preparing a thermally stable vaccine will obtain the great value and the market prospect.

So far, there are two main methods to improve the thermostability of the virus: (1) Introducing peptides on the virus surface that can induce self-biomineralization. For example, Wang et al. [18] integrated the biomimetic nucleating peptides that can induce calcium phosphate mineralization onto the capsid protein of enterovirus 71 (EV71), which increased the thermal stability of EV71 significantly. (2) Introducing rational amino acids into structural proteins that increase the interactions within viral protein subunits or between the capsid-nucleic acids. Mateo et al. [19] constructed two recombinant mutant strains of FMDV (A2065H and D3069E-T2188A) by reverse genetics, which proved that the two mutant viruses could increase the thermo-stability significantly, comparing with the parental virus. Kotecha et al. [20] constructed the FMDV serotype SAT2 mutant strain containing S2093Y, which showed the better thermo-stability than that of the original wild strain. Scott et al. [21] also showed that the mutation S2093H in serotype SAT2 recombinant virus could also improve the thermo-stability, and serotype SAT2 mutant strain containing $\mathrm{S} 2093 \mathrm{H}$ could be selected as a candidate for stabilizing SAT2 vaccine.

Here in this study, a full-length infectious clone of FMD type 0 marker virus with the excellent immunogenicity was used as the skeleton, and some key amino acids of FDMV structure protein related to the thermo-stability were mutated. Then, several recombinant strains were constructed and rescued. Furthermore, their biological characteristics, temperature and $\mathrm{pH}$ inactivating rates and immunogenicity were evaluated to assess their stability. To sum up, we reported that the rHN/DY-VP1Y2098F mutant carrying substitution Y2098F could be a candidate for developing an inactivated FMD vaccine with the enhanced thermostability.

\section{Materials And Methods}

\section{Cells, viruses, and plasmids}

BHK-21 (Baby hamster kidney clone 13 cells; strain 21; ATCC CCL-10) were maintained and propagated in minimal essential medium (MEM) with $10 \%$ fetal bovine serum (FBS, Gibco, Australia). BSR-T7/5 cells expressing T7RNA polymerase were obtained from Karl-Klaus Conzelmann (Max-von-Pettenkofer Institut, Munich, Germany) and were cultivated in Glasgow minimal essential medium (GMEM) supplemented containing 10\% FBS and 4\% tryptose phosphate broth, 10\% FBS. Besides, in alternate passages, $1 \mathrm{mg} / \mathrm{ml}$ G418 was added to ensure maintenance of the T7 polymerase gene. All cells were grown in a humidified chamber at $37^{\circ} \mathrm{C}$ supplemented with $5 \% \mathrm{CO}_{2}$. The plasmid pO/DY-VP1, a previously constructed type 0 FMDV full-length infectious clone which based on the constructed FMDV vaccine strain pOZK/93 - 08 [22], 3B1 and 3B2 were mutated $\left({ }^{4} A G P^{6}-4{ }^{4}{ }^{2} A^{6}\right)$ to eliminate the dominant epitope of 3B non-structural 
protein, served as the genetic backbone to construct some recombinant CDNA clones containing single or multiple capsid-stabilizing mutations in the structural protein. The virus recovered from pO/DY-VP1 is referred to as rHN/DY-VP1 (parental virus) which cannot react with monoclonal antibody 3B4B1.

\section{Identification of stabilizing residues and Generation of recombinant CDNA mutants}

Some recombinant virus mutants were designed in the plasmid pO/DY-VP1 (Fig. 1a) for studying the function of single or multiple mutations. According to the reported substitutions responsible for the thermostability [20,21], three mutants S2093F, S2093H and Y2098F were introduced in VP2. Additionally, V2090A was included in subsequent process because this substitution was suspected to help other introduced mutant for being passaged stably in cells. Five schemes were designed, containing S2093F, Y2098F, V2090A-S2093F, V2090A-S2093H and V2090A-S2093H-Y2098F. The site-directed mutagenesis were performed by a bio-tech company called GENEWIZ (Suzhou, China, www.genewiz.com.cn) and all the molecular constructs were performed by using standard molecular biological techniques [23]. All recombinant plasmids were verified by nucleotide sequencing and ensure that there was no other mutation occurring in the cloning process.

\section{Transfection and virus recovery}

The Not I-linearized recombinant plasmids were purified by a QIAquick PCR Purification Kit (Qiagen), and transfected into BSR/T7 cells monolayers with a Gene Pulser Xcell (two pulses at 150V and $950 \mathrm{uF}$ ) and Ingenio ${ }^{\circledR}$ Electroporation Kits (Mirus). The cells were further maintained at $37^{\circ} \mathrm{C}$ in growth medium. After 48-72 h, The BSR-T7 cells showed cytopathic effects (CPE) and were frozen at $-80^{\circ} \mathrm{C}$. After thawing and centrifugation, the virus-containing cell culture supernatants (named 'passage 0 ', P0) were serially passaged 8 times on BHK-21 cells. The total RNA was extracted from passage 8 supernatant of each rescued virus, and nucleotide sequencing verified that the mutated viruses derived from the genomelength cDNA (Fig. 1a).

\section{Plaque, growth kinetics and thermal inactivation assays}

Each plaque assays were performed in a 6-well plate and repeated 3 times. BHK-21 monolayer cells were infected with $200 \mathrm{uL}$ serial dilutions of viral samples. After $1 \mathrm{~h}$ incubation at $37^{\circ} \mathrm{C}$, the cells were overlaid with $2 \mathrm{~mL}$ gum tragacanth (MP Biomedicals). After incubating at $37^{\circ} \mathrm{C}$ for $48 \mathrm{~h}$, cells were fixed in $50 \%$ acetone and $50 \%$ methylalcohol and stained with $1 \%$ crystal violet, as previously described [24].

Growth kinetics of the virus was performed with plaque assays. Confluent monolayers of BHK-21 cells were infected with mutant viruses and parental virus at a multiplicity of infection (MOI) of 0.1 at $37^{\circ} \mathrm{C}$. After $1 \mathrm{~h}$ of adsorption, the inoculum was removed, and cells were washed with $0.01 \mathrm{M}$ phosphatebuffered saline (PBS; pH7.4) to remove unattached viruses. Then, cells were supplemented $2 \mathrm{~mL}$ complete medium and incubated at $37^{\circ} \mathrm{C}$. At 4, 8, 12 and 20 h.p.i., the virus-infected supernatants were collected and frozen at $-80^{\circ} \mathrm{C}$. Virus titers were calculated by plaque-forming units (PFU) $\mathrm{mL}^{-1}$, as described elsewhere [25]. 
Thermal inactivation was performed on parental and recombinant viruses cell culture supernatants which were uniformly diluted to $5 \times 10^{5} \mathrm{PFU} / \mathrm{mL}$ in TNE buffer (100 mM Tris, $\mathrm{pH} 7.4,10 \mathrm{mM}$ EDTA, $150 \mathrm{mM}$ $\mathrm{NaCl}$ ), completely as described previously [26]. After that, infectious particles were put into $42^{\circ} \mathrm{C}$ water bath for different times of heat treatment. To be specific, all viruses were incubated at temperatures of $42^{\circ} \mathrm{C}$ for $0,15,30,45,60,120,180,240 \mathrm{~min}$ and $49^{\circ} \mathrm{C}$ for $0,15,30,45,60 \mathrm{~min}$. Immediately after the heat treatment, these viruses were cooled onto the pre-prepared ice, and the viruses were titrated on BHK-21 cells by plaque assays. Each experiment was repeated twice. In addition, a pH inactivation kinetics assay was performed in TNE buffer $(\mathrm{pH}=6.0)$ with a steady temperature $\left(25^{\circ} \mathrm{C}\right)$ for $0,15,30,45,60,120 \mathrm{~min}$. The logarithmic values of all viruses titers at the different time points were linearly fitted respectively, then the slopes were calculated which could be used to determine the inactivation rate $[27,28]$. The percentage of infectious particles remaining was also computed and plotted [29], which was determined as the titer at each time point divided by the initial titer $(0 \mathrm{~min}) \times 100 \%$.

\section{Virus inactivation, Purification}

The eighth passages of parental and mutants' viruses were expanded and cultured on BHK-21 cells. After being freezed-thawed for 3 times, the cells were centrifuged at $4^{\circ} \mathrm{C}$ at $1500 \times \mathrm{g}$ for $30 \mathrm{~min}$ to remove the cell fragments and collect the supernatant and lysates. Clarified supernatant was made $1.2 \%$ in binary ethyleneimine (BEI) and inactivated at $30^{\circ} \mathrm{C}$ for $28 \mathrm{~h}$ [30]. During the inactivation period, the mixture is reversed and mixed per hour. Inactivated virus was inoculated into 2-day-old suckling mice to test whether the virus was inactivated completely [31].

Inactivated viruses were concentrated with $80 \mathrm{~mL} / \mathrm{L}$ polyethylene glycol 8000 (PEG 8000; Sigma-Aldrich) and purified on $10 \mathrm{~g} / \mathrm{mL}$ to $50 \mathrm{~g} / \mathrm{mL}$ sucrose density gradients (SDG), and the mixture was centrifuged at $4^{\circ} \mathrm{C}, 104000 \times \mathrm{g}$ for $3 \mathrm{~h}$. Following fractionation, $146 \mathrm{~S}$ were collected by measuring the absorbance at $260 \mathrm{~nm}$. The collected $146 \mathrm{~S}$ was centrifuged at $130000 \times \mathrm{g}$ for $4 \mathrm{~h}$ at $4^{\circ} \mathrm{C}$ to remove sucrose, and the supernatant was discarded. The precipitation was then resuspended with $200 \mathrm{uL}$ TNE buffer $(50 \mathrm{mM}$ Tris/HCL [pH 7.4], $10 \mathrm{mM}$ EDTA, $150 \mathrm{mM} \mathrm{NaCl}$ ) [26].

\section{Immunofluorescent assay}

BHK-21 cells $\left(2 \times 10^{5}\right)$ grown on a six-well plate were infected with parental and mutant virus at a $\mathrm{MOI}$ of 1 respectively. At $5 \mathrm{~h}$ post-infection (p.i.), the cells were fixed with $4 \%$ paraformaldehyde for $30 \mathrm{~min}$ at $4^{\circ} \mathrm{C}$. The cells were washed three times and permeabilized for 10 min with $0.5 \%$ Triton X-100 in PBS and blocked for $1 \mathrm{~h}$ with $5 \mathrm{~g} / \mathrm{mL}$ BAS bovine serum albumin in PBS. Then, the cells were washed with PBS 3 times and incubated for $1 \mathrm{~h}$ with MAb 3A24 or 3B4B1 (MAb 3A24 directed against AEKNPLE (residues 99-105) epitope in NSP(Non-SP) 3A of FMDV and MAb 3B4B1 directed against GPYAGPMER (residues 1-9) epitope in NSP 3B2 were obtained from the Lanzhou Veterinary Research Institute (LVRI)), then the cells were washed 5 times and stained with fluorescein isothiocyanate (FITC)-conjugated goat antimouse IgG antibody (purchased from Sigma) for another $1 \mathrm{~h}$. The cells were observed in a Leica DMI6000B fluorescence microscope.

\section{Animal experiments}


The animal experiments were performed under Biosafety Level 3 conditions in the animal facilities at LVRI following the protocol approved by the Review Board of LVRI, Chinese Academy of Agricultural Sciences (Permission number: SYXK-GAN-2004-0005). At the beginning of the experiments, all animals were negative for FMDV specific antibodies. The animals were euthanized by intravenous injection of sodium pentobarbital at the end of all experiments.

Briefly, sixteen 3-month-old pigs were divided into 4 groups which were kept in a separate room respectively. Pigs in group 1 were inoculated with the vaccine containing inactivated parental virus, and which in group 2-4 were inoculated with the vaccine containing inactivated mutant virus separately. Each animal received $3 \mu \mathrm{g}$ of purified $146 \mathrm{~S}$ antigen formulated in water-in-oil-in-water emulsions with Montanide ISA 201 (Seppic, Paris, France) by intramuscular injecting on days 0. Furthermore, serum samples collected at 7, 14, 21, 28, 35 and 42 dpi were tested by virus neutralizing-antibody titers (VNT) as previously described [32].

For the guinea-pig experiments, inactivated parental and stabilized mutant viruses were emulsified with ISA201 adjuvant, then 40 guinea pigs, aged 2 months, were divided into 4 groups ( $n=10$ per group). Group 1 was inoculated with the vaccine which contained inactivated parental virus and was stored for 4 months at $4^{\circ} \mathrm{C}$, and group 2-4 was inoculated with the vaccines which contained inactivated mutant virus respectively and were stored for 4 months at $4^{\circ} \mathrm{C}$. Each guinea pig received $0.5 \mu \mathrm{g}$ of purified $146 \mathrm{~S}$ antigen by intramuscular injection. Furthermore, serum samples collected at $28 \mathrm{dpi}$ were tested by virus neutralizing-antibody titers (VNT) as previously described [32]. The experiments were repeated with the same volumes after the vaccines had been stored for 6 months at $4^{\circ} \mathrm{C}$.

No statistical method was used to predetermine sample size. Group sizes for the pigs and guinea-pigs immunization studies were consistent with those in previously published studies [33,34].

\section{Titration of neutralizing antibodies}

The sera from guinea pigs and pigs were prepared from blood samples. Neutralizing-antibody titers were calculated by the Spearmann-Karber method, and were expressed as the reciprocal of the highest serum dilution neutralizing $50 \%$ of $100 \mathrm{TCID}_{50}$ of the homologous virus [35]. The mean antibody titers of each group were compared with threshold antibody titers that have previously been shown to correlate with protection $[33,34]$.

\section{Date analysis}

All statistical analyses and the graphs were carried out using GraphPad Prism v5.0 (GraphPad Software). $\mathrm{P}<0.05$ was considered statistically significant.

\section{Results}

\section{Design of mutation and generation of the recombinant viruses}


To establish the effects of the individual or the multiple amino acids substitutions in the FMDV's structural proteins (SP), we constructed the recombinant virus mutants in an infectious, genome-length clone of FMDV, pO/DY-VP1 (Fig. 1a). We introduced the following amino acids substitutions into the VP2 coding region: S2093F, S2093H and Y2098F, which were responsible for the resistance to heat [20,21]. At first, we just introduced S2093F substitution into the infectious clone pO/DY-VP1, and the constructed plasmids were termed as $\mathrm{pO} / \mathrm{DY}$-VP1 $1^{\mathrm{S} 2093 \mathrm{~F}}$. The therefore achieved infectious mutant viral strain named as $\mathrm{rHN} / \mathrm{DY}-\mathrm{VP} 1^{\mathrm{S} 2093 \mathrm{~F}}$ was obtained by the transfection of the corresponding linearized plasmids into BSR/T7 cells and passaged in BHK-21 cells. After eight consecutive passages (P8), the sequence analysis illustrated that some of the rHN/DY-VP1 ${ }^{\text {S2093F }}$ strain containing S2093F were reversed, but the rest of the rHN/DY-VP1 ${ }^{\text {S2093F }}$ containing the expected substitution S2093F gained an extra rHN/DYVP1 ${ }^{\text {S2093F }}$ mutation V2090A during the passage. The reason for that phenomenon is that some compensatory mutations prone to occur, contributing to the increasing genetic stability in some virus passage studies [36]. It was speculated that the presence of V2090A substitution could increase the stable inheritance of other introduced thermostable amino acids. As a result, a mutation of V2090A was introduced into some of the later designed constructs. Eventually, four mutants Y2098F, V2090A-S2093F, V2090A-S2093H and V2090A-S2093H-Y2098F were constructed, and four infectious mutant viral strains were obtained and sequenced after eight consecutive passages (P8). The results illustrated that the four mutant viral strains had been expected, with no other mutations found. The parental and mutant viral strains were analyzed with the indirect immunofluorescence assay. The testing results showed that the recombinant viral strains have the skeleton of parental virus rHN/DY-VP1 (Fig. 1b), indicating that the 4

recombinant strains were rescued successfully and named rHN/DY-VP1 ${ }^{\text {Y2098F, }}$, rHN/DY-VP1 V2090A - S2093F, rHN/DY-VP1 V2090A - S2093H and rHN/DY-VP1V2090A - S2093H-Y2098F respectively. Furthermore, recombinant strains were verified by Negative-stain electron microscopy. The assay revealed that the clear and the intact virions of the recombinant strains, whose morphological structure and the same size (around $25 \mathrm{~nm}$ ) identical to the parental virus [37].

\section{Infectivity and growth characteristics of the engineered mutants}

As shown in Fig. 2a, the plaque morphologies of the rescued strain rHN/DY-VP1 ${ }^{\mathrm{Y} 2098 \mathrm{~F}}$, rHN/DYVP1 V2090A - S2093F, rHN/DY-VP1 V2090A - S2093H and rHN/DY-VP1 V2090A - S2093H-Y2098F were formed on BHK-21 cells and were similar to that of the control virus rHN/DY-VP1, showing that the introduction of the thermostable mutations had little influence on the infectivity of the recombinant strains.

In order to explore the growth properties of the parental and the four mutant viruses in more detail, growth kinetics were assayed in BHK-21 monolayer cells. The assay results in Fig. $2 \mathrm{~b}$ showed that the titer at 4$8 \mathrm{~h} \mathrm{p.i.} \mathrm{of} \mathrm{the} 4$ rescue strains were similar to that of the parental control virus $(p>0.05)$. After the cell was infected for $12 \mathrm{~h}$ with different viral strains, the titers of $\mathrm{rHN} / \mathrm{DY}-\mathrm{VP} 1^{\mathrm{Y} 2098 \mathrm{~F}}$ and $\mathrm{rHN} / \mathrm{DY}-\mathrm{VP} 1^{\mathrm{V} 2090 \mathrm{~A}-}$ S2093H-Y2098F were similar to that of the parental rHN/DY-VP1 $(p>0.05)$; while the titers of saved rHN/DYVP1 $12090 \mathrm{~A}$ - S2093F and rHN/DY-VP1 V2090A - S2093H were significantly lower than that of rHN/DY-VP1 ( $p<$ 
0.05). The possibly reason for that maybe the over-stabilization hinders during the release of the viral genome during the viral cell entry. At 20 h.p.i, the titers of the 4 recombinant strains were similar to that of their parental strain rHN/DY-VP1 $(p>0.05)$. In conclusion, the final results indicated that the replication capacity of 4 recombinant viral strains were similar to that of parental virus on the whole.

\section{Thermal and acid stabilities of the mutants determined by thermal inactivation assay}

The inactivation rate and the residual infectious particles of the virus were assayed after the temperature treatment, the key indicator of thermal stability. The rescued viral strains and the parental virus were uniformly diluted with titers of $5 \times 10^{5} \mathrm{PFU} / \mathrm{mL}$ and were either treated at $\mathrm{pH} 6.0$ or heated at $42^{\circ} \mathrm{C}$ or $49^{\circ} \mathrm{C}$ for different times, up to $4 \mathrm{~h}$ (Fig. 3). The inactivation rate of the parental virus and mutant strains at $42^{\circ} \mathrm{C}$ or $49^{\circ} \mathrm{C}$ was obtained by calculating the slope of linear kinetics. The lower inactivation rate means that the virus is more stable.

At $42^{\circ} \mathrm{C}$, the inactivation rate of parental viral strain rHN/DY-VP1 was $0.0052 \mathrm{~min}^{-1}$; and the percentage of the remaining infectious particles was $4.4 \%$ after 240 min treatment (Fig. 3a and $3 \mathrm{~b}$ ). As for mutant strains rHN/DY-VP1 ${ }^{\text {Y2098F, }}$, rHN/DY-VP1 V2090A - S2093F, rHN/DY-VP1 V2090A - S2093H and rHN/DY-VP1 V2090A - $\mathrm{S} 2093 \mathrm{H}-\mathrm{Y} 2098 \mathrm{~F}$, the inactivation rates were $0.0044,0.0054,0.0049$, and $0.0045 \mathrm{~min}^{-1}$, respectively, and the percentage of the infectious particles remaining were $8.6 \%, 4.8 \%, 8.0 \%$ and $7.4 \%$, respectively (Fig. $3 a$ and $3 b)$. The data showed that the inactivation rates of rHN/DY-VP1 ${ }^{\mathrm{Y} 2098 \mathrm{~F}}, \mathrm{rHN} / \mathrm{DY}-\mathrm{VP} 1^{\mathrm{V} 2090 \mathrm{~A}-\mathrm{S} 2093 \mathrm{H}}$ and rHN/DY-VP1 V2090A - S2093H-Y2098F were lower than that of the parental strain rHN/DY-VP1, and the capsids of these three recombinant viruses depolymerized more slowly at $42^{\circ} \mathrm{C}$.

The infectious viral particles were tested at $49^{\circ} \mathrm{C}$ for $1 \mathrm{~h}$ for further study of the thermo-stability of the mutant FMDV strains. The inactivation rate of parental virus rHN/DY-VP1 was $0.014 \mathrm{~min}^{-1}$, and the remaining percentage of the original infectious viral particles was $15.4 \%$ after 60 min treatment (Fig. 3c and 3d), but the inactivation rates of the mutant viral strains rHN/DY-VP1Y2098F, rHN/DY-VP1 V2090A S2093F, rHN/DY-VP1 V2090A - S2093H and rHN/DY-VP1 V2090A - S2093H-Y2098F were 0.009, 0.039, 0.031 and $0.008 \mathrm{~min}^{-1}$, respectively. The remaining percentage of the infectious particles of those 4 types of mutant viral strains were $32 \%, 0.35 \%, 0.88 \%$ and $35 \%$, respectively (Fig. $3 \mathrm{c}$ and $3 \mathrm{~d}$ ). The testing data showed that the inactivation rates of rHN/DY-VP1 ${ }^{\mathrm{Y} 2098 \mathrm{~F}}$, and rHN/DY-VP1 V2090A - S2093H-Y2098F were lower than that of parental strain rHN/DY-VP1, and the capsids of those two recombinant viral strains depolymerized more slowly at $49^{\circ} \mathrm{C}$.

In order to test the stability of recombinant virus in the acid environment, the acid inactivation assay was conducted at $\mathrm{pH} 6.0$ in the room temperature. The assay results showed that, comparing to that of the parental virus $\left(0.0038 \mathrm{~min}^{-1}\right)$, those mutations improved $\mathrm{pH}$ stability for the mutated viral strains rHN/DY-VP1 ${ }^{\mathrm{Y} 2098 \mathrm{~F}}\left(0.0028 \mathrm{~min}^{-1}\right)$, rHN/DY-VP1 V2090A - S2093F $\left(0.003 \mathrm{~min}^{-1}\right)$, rHN/DY-VP1 V2090A - S2093H $\left(0.0022 \mathrm{~min}^{-1}\right)$, and rHN/DY-VP1 V2090A - S2093H-Y2098F $\left(0.0035 \mathrm{~min}^{-1}\right)$ (Fig. 3e). The remaining 
percentages of infectious particles after $2 \mathrm{~h}$ treatment at $\mathrm{pH}$ 6.0, of mutant viral strains the rHN/DYVP1 ${ }^{\mathrm{Y} 2098 \mathrm{~F}}$, rHN/DY-VP1 V2090A - S2093F, rHN/DY-VP1 V2090A - S2093H and rHN/DY-VP1 V2090A - S2093H-Y2098F were $44.4 \%, 35.6 \%, 46 \%$ and $38.0 \%$, respectively, higher than $22.6 \%$ comparing to the parental virus rHN/DY-VP1 (Fig. 3f).

\section{Immunogenicity of stabilized mutants}

The virus neutralization test was carried out to analyze whether heat-resistant mutations would affect the immunogenicity of the parental virus. The results shown in Fig. 4a, that all pigs were vaccinated after the parental strain rHN/DY-VP1 and the mutant strains rHN/DY-VP1 ${ }^{\text {Y2098F, }}$, rHN/DY-VP1 ${ }^{\text {V2090A - S2093H }}$ and rHN/DY-VP1 V2090A - S2093H-Y2098F were made into vaccines. The blood samples were collected and neutralizing tested every 7 days until the 7 th week. The protective neutralizing antibody titers (VNTs) produced by each types of potential viral vaccine strains were calculated and plotted using the average data of each group. The results of statistical analysis showed that the levels of neutralizing antibodies produced by rHN/DY-VP1 Y2098F, rHN/DY-VP1 V2090A - S2093H and rHN/DY-VP1V2090A - S2093H-Y2098F potential vaccine strains were roughly identical to that of the parental rHN/DY-VP1 vaccine strain $(P>$ 0.05). At the same time, the data indicated that the high levels of protective neutralizing antibodies (VNT $\geq 2 \log _{10}$ ) provided the protection for the pigs on day 35 after immunization of the potential vaccine strains [33].

The immunogenicity was further compared after long-term storage at $4^{\circ} \mathrm{C}$ to study the stability of the potential thermo-stable recombinant vaccine strains. We stored the potential parental and recombinant viral vaccine strains equally at $4^{\circ} \mathrm{C}$ for 4 months, which we then used to vaccinate two groups of the guinea pigs, ten of them in each group. The titers of the protective neutralizing antibody produced by each potential vaccine stain were calculated and plotted for comparing the average data of each group (Fig. 4b). The data comparison showed that only potential rHN/DY-VP1 ${ }^{\mathrm{Y} 2098 \mathrm{~F}}$ vaccine strain produced significantly higher titer of the neutralizing antibodies than the parental rHN/DY-VP1 strain $(P<0.05)$. The potential recombinant $\mathrm{rHN} / \mathrm{DY}$-VP1 ${ }^{\mathrm{Y} 2098 \mathrm{~F}}$ vaccine strain showed the better stability than the potential parental vaccine strain rHN/DY-VP1.

In a further iteration, we stored the parental and recombinant vaccine strains equally at $4^{\circ} \mathrm{C}$ for 6 months, which we then used to immunize two groups of the guinea pigs, ten of them in each group (Fig. 4c). The data comparison showed that only the potential rHN/DY-VP1 ${ }^{\mathrm{Y} 2098 \mathrm{~F}}$ vaccine strain produced significantly higher neutralizing antibodies than the parental rHN/DY-VP1 $(P<0.05)$. The potential recombinant rHN/DY-VP1 ${ }^{\text {Y2098F }}$ vaccine strain showed the better stability than the potential parental vaccine strain rHN/DY-VP1.

\section{Discussion}

Although the present conventional FMD vaccines can prevent clinically, the protection period is short lived, normally approximately 6 months [10-12]. Therefore, if the duration of the vaccine strain's stability 
could be increased, the FMD vaccines could have the potential to be more durable and more protective. At present, studies on the thermal stability of the capsids of FMDV still meet some difficulties [21]. The thermal stability of FMDV can be influenced by the disulphide bridges, electrostatic interactions, hydrogen bonds, and salt bridges of the viral capsids $[20,38,39]$. Mateo and his colleagues suggested that the disulfide bonds or the electrostatic interactions between the viral subunits could be used to increase the FMDV's thermostability, which can be an effective tool for development of the better vaccines [19]. We designed the mutation scheme and experimental scheme here by referring to the previous studies and the described mutation sites of the FMDV's structural proteins [20]. With this research, we hope to screen out the strains with the excellent characteristics and the thermal stability to provide the improvement for the FMDV's inactivated vaccines.

Based on the bonding energies in silico [18], 5 mutations were eventually designed. Since VP2 93 is an important site, each of the 5 mutation schemes contains the VP2 93 mutation. The S2093H and Y2098F substitutions were used together to form a double-mutated sites mutant strain, which is better comparing to the mutants strains containing only a single heat-resistant mutation. A new amino acid mutation, V2090A, emerged during the viral passage, which we suspected a compensatory mutation contributing to the stable inheritance of VP2 93 substitution.

The experiments of biological characteristics showed that the titers of the viral strains containing S2093F and $\mathbf{S} 2093 \mathrm{H}$ mutation at $12 \mathrm{~h}$.p.i, were lower than that of the parental strain. It is possible that overstabilization leads to poor uncoating and replication of FMDV. 0 'donnell et al. found that the effective release of viral RNA in cells is necessary for FMDV replication [40].

Based on the free energy values of FMDV's binding published by Kotecha and colleagues [16], S2093F was more stable than $\mathrm{S} 2093 \mathrm{H}$ and $\mathrm{Y} 2098 \mathrm{~F}$, but our experiments showed that for the increase of the thermal stability, the Y2098F and the S2093H-Y2098F mutated strains were better than the S2093H. It was also proved in our study here that the $\mathrm{Y} 2098 \mathrm{~F}$ mutation was better than $\mathrm{S} 2093 \mathrm{H}$ mutation to increasing FMDV's thermal stability. Similarly reported that the Y2098F substation strain is more thermally stable with the FMDV's serotype VLP strains [41]. Our testing results relevant to the viral thermal stability proved that the three mutation modes of S2093H, Y2098F and S2093H-Y2098F not only had no lethal effect on the mutated viral strains, but also could increase the thermo-stability of FMDV's serotype 0 strains.

FMD's virions are not only extremely thermolabile but also remarkably acid labile. FMDV's acid sensitivity is mainly regulated by electrostatic interactions and hydrogen bonds formed by the side chain of amino acid residues in the viral capsid [42]. Because there are some similarities in the determining factors of FMDV's thermo-stability and its acid stability, it is possible that a heat-resistant FMDV strain can also has the increased resistance to the acid inactivation treatment. In this study, the acid inactivation experiments proved that rHN/DY-VP1 ${ }^{\text {Y2098F, }}$, rHN/DY-VP1 V2090A - S2093H and rHN/DY-VP1 V2090A - S2093H-Y2098F strains had not only the heat resistant properties but also the acid stabilities. 
In order to further evaluate the potentiality of the heat-resistant recombinant viral strains as a possible candidate strains for the inactivated FMD vaccine, the immunogenicity of recombinant viral strains was investigated. The results here showed that rHN/DY-VP1 ${ }^{\mathrm{Y} 2098 \mathrm{~F}}$, rHN/DY-VP1 V2090A-S2093H and rHN/DYVP1 V2090A - S2093H-Y2098F strains had the similar immunogenicity with the parental rHN/DY-VP1 strain, indicating that the Y2098F, S2093H and S2093H-Y2098F mutations did not influence the immunogenicity of the 0 serotype FMDV. Meanwhile, guinea pigs were further immunized with the potential vaccine strains prepared by the aforementioned recombinant strains and the parental strains at $4^{\circ} \mathrm{C}$ for different periods of time to analyze the differences in the immune response in the body, induced by the vaccination. The results here showed that after 4 or 6 months of refrigerated at $4{ }^{\circ} \mathrm{C}$, the vaccine prepared by $\mathrm{rHN} / \mathrm{DY}-\mathrm{VP} 1^{\mathrm{Y} 2098 \mathrm{~F}}$ recombinant strain induced the animal to produce the higher neutralizing antibodies comparing to the parental virus $(P<0.05)$, indicating that the Y2098F mutation could significantly increase the thermal stability of the potential vaccine strain.

In particular, the titer of neutralizing antibody produced by the recombinant strain in the animal body in this study was twice as high as that of the parental viral strain, although it is not as high as previously reported in the literature [20], which may be due to the lower dose of antigen inoculated when the animals were vaccinated.

In conclusion, the rHN/DY-VP1 ${ }^{\mathrm{Y} 2098 \mathrm{~F}}$ recombinant heat-resistant strain can act as a kind of potential vaccine candidate. The $\mathrm{rHN} / \mathrm{DY}-\mathrm{VP} 1^{\mathrm{Y} 2098 \mathrm{~F}}$ strain is not only thermally stable and $\mathrm{pH}$-stable, but also similar to its parental strain in the infectivity and the replication. More importantly, the immunogenicity of the recombinant strain was not influenced by the mutations of the amino acids aforementioned, and the $\mathrm{rHN} / \mathrm{DY}-\mathrm{VP} 1^{\mathrm{Y} 2098 \mathrm{~F}}$ recombinant vaccine strain was more stable than the parental viral strain. We believe that the rHN/DY-VP1 ${ }^{\mathrm{Y} 2098 \mathrm{~F}}$ could be selected as the potential effective FMD type 0 vaccines with the better thermo-stabilities in the future.

\section{Declarations}

\section{Funding}

This work was supported by National Key Research and Development Programs (2017YFD0500902) \& Gansu Youth Science and Technology Fund Project (1606RJYA256).

\section{Conflict of interest}

The authors declare that they have no conflict of interest.

\section{References}


1. Alexandersen S, Zhang Z, Donaldson Al, Garland AJ (2003) The pathogenesis and diagnosis of footand-mouth disease. J Comp Pathol 129 (1):1-36. doi:https://doi.org/10.1016/s0021-9975(03)000410

2. Brown F (2003) The history of research in foot-and-mouth disease. Virus research 91 (1):3-7. doi:https://doi.org/10.1016/s0168-1702(02)00268-x

3. Sobrino F, Saiz M, Jimenez-Clavero MA, Nunez JI, Rosas MF, Baranowski E, Ley V (2001) Foot-andmouth disease virus: a long known virus, but a current threat. Vet Res 32 (1):1-30. doi:https://doi.org/10.1051/vetres:2001106

4. Doel TR, Baccarini PJ (1981) Thermal stability of foot-and-mouth disease virus. Archives of virology 70 (1):21-32. doi:https://doi.org/10.1007/BF01320790

5. Doel TR, Chong WK (1982) Comparative immunogenicity of 146S, 75 S and $12 S$ particles of foot-andmouth disease virus. Arch Virol 73 (2):185-191. doi:https://doi.org/10.1007/BF01314726

6. Curry S, Abrams CC, Fry E, Crowther JC, Belsham GJ, Stuart DI, King AM (1995) Viral RNA modulates the acid sensitivity of foot-and-mouth disease virus capsids. J Virol 69 (1):430-438. doi:https://doi.org/10.1128/JVI.69.1.430-438.1995

7. Newman JF, Rowlands DJ, Brown F (1973) A physico-chemical sub-grouping of the mammalian picornaviruses. J Gen Virol 18 (2):171-180. doi:https://doi.org/10.1099/0022-1317-18-2-171

8. Vázquez-Calvo A, Caridi F, Sobrino F, Martín-Acebes MA (2014) An increase in acid resistance of footand-mouth disease virus capsid is mediated by a tyrosine replacement of the VP2 histidine previously associated with VP0 cleavage. Journal of virology 88 (5):3039-3042. doi:https://doi.org/10.1128/JVI.03222-13

9. Kitching P, Hammond J, Jeggo M, Charleston B, Paton D, Rodriguez L, Heckert R (2007) Global FMD control-is it an option? Vaccine 25 (30):5660-5664. doi:https://doi.org/10.1016/j.vaccine.2006.10.052

10. Hunter $P$ (1996) The performance of southern African territories serotypes of foot and mouth disease antigen in oil-adjuvanted vaccines. Rev Sci Tech 15 (3):913-922. doi:https://doi.org/10.20506/rst.15.3.954

11. Parida S (2009) Vaccination against foot-and-mouth disease virus: strategies and effectiveness. Expert Rev Vaccines 8 (3):347-365. doi:https://doi.org/10.1586/14760584.8.3.347

12. Cox SJ, Aggarwal N, Statham RJ, Barnett PV (2003) Longevity of antibody and cytokine responses following vaccination with high potency emergency FMD vaccines. Vaccine 21 (13-14):1336-1347. doi:https://doi.org/10.1016/s0264-410x(02)00691-6

13. Doel TR (2003) FMD vaccines. Virus Res 91 (1):81-99. doi:https://doi.org/10.1016/s01681702(02)00261-7

14. Hall MD, Knowles NJ, Wadsworth J, Rambaut A, Woolhouse ME (2013) Reconstructing geographical movements and host species transitions of foot-and-mouth disease virus serotype SAT 2. mBio 4 (5):e00591-00513. doi:https://doi.org/10.1128/mBio.00591-13 
15. Schlehuber LD, McFadyen IJ, Shu Y, Carignan J, Duprex WP, Forsyth WR, Ho JH, Kitsos CM, Lee GY, Levinson DA, Lucier SC, Moore CB, Nguyen NT, Ramos J, Weinstock BA, Zhang J, Monagle JA, Gardner CR, Alvarez JC (2011) Towards ambient temperature-stable vaccines: the identification of thermally stabilizing liquid formulations for measles virus using an innovative high-throughput infectivity assay. Vaccine 29 (31):5031-5039. doi:https://doi.org/10.1016/j.vaccine.2011.04.079

16. Chen X, Fernando GJ, Crichton ML, Flaim C, Yukiko SR, Fairmaid EJ, Corbett HJ, Primiero CA, Ansaldo $A B$, Frazer IH, Brown LE, Kendall MA (2011) Improving the reach of vaccines to low-resource regions, with a needle-free vaccine delivery device and long-term thermostabilization. Journal of controlled release : official journal of the Controlled Release Society 152 (3):349-355. doi:https://doi.org/10.1016/j.jconrel.2011.02.026

17. Das P (2004) Revolutionary vaccine technology breaks the cold chain. Lancet Infect Dis 4 (12):719. doi:https://doi.org/10.1016/s1473-3099(04)01222-8

18. Wang G, Cao RY, Chen R, Mo L, Han JF, Wang X, Xu X, Jiang T, Deng YQ, Lyu K, Zhu SY, Qin ED, Tang $R$, Qin CF (2013) Rational design of thermostable vaccines by engineered peptide-induced virus selfbiomineralization under physiological conditions. Proceedings of the National Academy of Sciences of the United States of America 110 (19):7619-7624. doi:https://doi.org/10.1073/pnas.1300233110

19. Mateo R, Luna E, Rincon V, Mateu MG (2008) Engineering viable foot-and-mouth disease viruses with increased thermostability as a step in the development of improved vaccines. J Virol 82 (24):1223212240. doi:https://doi.org/10.1128/JVI.01553-08

20. Kotecha A, Seago J, Scott K, Burman A, Loureiro S, Ren J, Porta C, Ginn HM, Jackson T, Perez-Martin E, Siebert CA, Paul G, Huiskonen JT, Jones IM, Esnouf RM, Fry EE, Maree FF, Charleston B, Stuart DI (2015) Structure-based energetics of protein interfaces guides foot-and-mouth disease virus vaccine design. Nat Struct Mol Biol 22 (10):788-794. doi:https://doi.org/10.1038/nsmb.3096

21. Scott KA, Kotecha A, Seago J, Ren J, Fry EE, Stuart DI, Charleston B, Maree FF (2017) SAT2 Foot-andMouth Disease Virus Structurally Modified for Increased Thermostability. Journal of virology 91 (10):e02312-e02316. doi:https://doi.org/10.1128/JVI.02312-16

22. Cao WJ, Li PH, Bai XW, Lu ZJ, Sun P, Liu ZX (2010) Rescue and Identification of Virus Activity of Foot-and-Mouth Disease Virus Strain O/HN/93 from Full-length cDNA Clone. ACTA AGRICULTURAE BOREALI-SINICA 25 (3):32-37. doi:http://doi.org/10.7668/hbnxb.2010.03.008

23. Sambrook J, Fritsch EF, Maniatis T (1982) Molecular Cloning: A Laboratory Manual. Cold Spring Harbor Laboratory, New York

24. Rieder E, Bunch T, Brown F, Mason PW (1993) Genetically engineered foot-and-mouth disease viruses with poly $(C)$ tracts of two nucleotides are virulent in mice. J Virol 67 (9):5139-5145. doi:https://doi.org/10.1128/JVI.67.9.5139-5145.1993

25. Pacheco JM, Henry TM, O'Donnell VK, Gregory JB, Mason PW (2003) Role of Nonstructural Proteins $3 \mathrm{~A}$ and $3 \mathrm{~B}$ in Host Rangeand Pathogenicity of Foot-and-Mouth DiseaseVirus. Journal of Virology 77 (24):13017-13027. doi:https://doi.org/10.1128/jvi.77.24.13017-13027.2003 
26. Knipe T, Rieder E, Baxt B, Ward G, Mason PW (1997) Characterization of synthetic foot-and-mouth disease virus provirions separates acid-mediated disassembly from infectivity. J Virol 71 (4):28512856. doi:https://doi.org/10.1128/JVI.71.4.2851-2856.1997

27. Mateo R, Luna E, Mateu MG (2007) Thermostable variants are not generally represented in foot-andmouth disease virus quasispecies. J Gen Virol 88 (Pt 3):859-864.

doi:https://doi.org/10.1099/vir.0.82521-0

28. Mateo R, Mateu MG (2007) Deterministic, compensatory mutational events in the capsid of foot-andmouth disease virus in response to the introduction of mutations found in viruses from persistent infections. J Virol 81 (4):1879-1887. doi:https://doi.org/10.1128/JVI.01899-06

29. Mateo R, Diaz A, Baranowski E, Mateu MG (2003) Complete alanine scanning of intersubunit interfaces in a foot-and-mouth disease virus capsid reveals critical contributions of many side chains to particle stability and viral function. J Biol Chem 278 (42):41019-41027. doi:https://doi.org/10.1074/jbc.M304990200

30. Fowler VL, Bashiruddin JB, Maree FF, Mutowembwa P, Bankowski B, Gibson D, Cox S, Knowles N, Barnett PV (2011) Foot-and-mouth disease marker vaccine: cattle protection with a partial VP1 G-H loop deleted virus antigen. Vaccine 29 (46):8405-8411. doi:https://doi.org/10.1016/j.vaccine.2011.08.035

31. OIE (2012) FMD. In: Manual of Standard for Diagnostic Test and Vaccine. OIE, Paris, pp 77-92

32. Shao J-J, Wong CK, Lin T, Lee SK, Cong G-Z, Sin FWY, Du J-Z, Gao S-D, Liu X-T, Cai X-P, Xie Y, Chang H-Y, Liu J-X (2011) Promising Multiple-Epitope Recombinant Vaccine against Foot-and-Mouth Disease Virus Type 0 in Swine. Clinical and Vaccine Immunology 18 (1):143-149. doi:https://doi.org/10.1128/CVI.00236-10

33. Barnett PV, Statham RJ, Vosloo W, Haydon DT (2003) Foot-and-mouth disease vaccine potency testing: determination and statistical validation of a model using a serological approach. Vaccine 21 (23):3240-3248. doi:https://doi.org/10.1016/s0264-410x(03)00219-6

34. Bolwell C, Parry NR, Rowlands DJ (1992) Comparison between in vitro neutralization titres and in vivo protection against homologous and heterologous challenge induced by vaccines prepared from two serologically distinct variants of foot-and-mouth disease virus, serotype A22. Journal of General Virology 73 (3):727-731. doi:https://doi.org/10.1099/0022-1317-73-3-727

35. Brehm KE, Ferris NP, Lenk M, Riebe R, Haas B (2009) Highly Sensitive Fetal Goat Tongue Cell Line for Detection and Isolation of Foot-and-Mouth Disease Virus. Journal of Clinical Microbiology 47 (10):3156-3160. doi:https://doi.org/10.1128/JCM.00510-09

36. Luongo C, Winter CC, Collins PL, Buchholz UJ (2012) Increased genetic and phenotypic stability of a promising live-attenuated respiratory syncytial virus vaccine candidate by reverse genetics. J Virol 86 (19):10792-10804. doi:https://doi.org/10.1128/JVI.01227-12

37. Schulze P, Olechnowitz AF (1975) Electron microscopy studies on the proliferation of foot-and-mouth disease virus in cell cultures. III. Morphogenesis in cytoplasm. Arch Exp Veterinarmed 29 (3):441-457 
38. Loladze VV, Ibarra-Molero B, Sanchez-Ruiz JM, Makhatadze GI (1999) Engineering a thermostable protein via optimization of charge-charge interactions on the protein surface. Biochemistry 38 (50):16419-16423. doi:https://doi.org/10.1021/bi992271w

39. Rincón V, Rodríguez-Huete A, López-Argüello S, Ibarra-Molero B, Sanchez-Ruiz Jose M, Harmsen Michiel M, Mateu Mauricio G (2014) Identification of the Structural Basis of Thermal Lability of a Virus Provides a Rationale for Improved Vaccines. Structure 22 (11):1560-1570. doi:https://doi.org/10.1016/j.str.2014.08.019

40. O'Donnell V, LaRocco M, Duque H, Baxt B (2005) Analysis of Foot-and-Mouth Disease Virus Internalization Events in Cultured Cells. Journal of Virology 79 (13):8506-8518. doi:https://doi.org/10.1128/JVI.79.13.8506-8518.2005

41. Ganji VK, Biswal JK, Lalzampuia H, Basagoudanavar SH, Saravanan P, Tamil Selvan RP, Umapathi V, Reddy GR, Sanyal A, Dechamma HJ (2018) Mutation in the VP2 gene of P1-2A capsid protein increases the thermostability of virus-like particles of foot-and-mouth disease virus serotype 0 . Applied Microbiology and Biotechnology 102 (20):8883-8893. doi:https://doi.org/10.1007/s00253018-9278-9

42. Yuan H, Li P, Bao H, Sun P, Bai X, Bai Q, Li N, Ma X, Cao Y, Fu Y, Li K, Zhang J, Li D, Chen Y, Zhang J, Lu Z, Liu Z (2020) Engineering viable foot-and-mouth disease viruses with increased acid stability facilitate the development of improved vaccines. Applied Microbiology and Biotechnology 104 (4):1683-1694. doi:https://doi.org/10.1007/s00253-019-10280-9

\section{Figures}


a

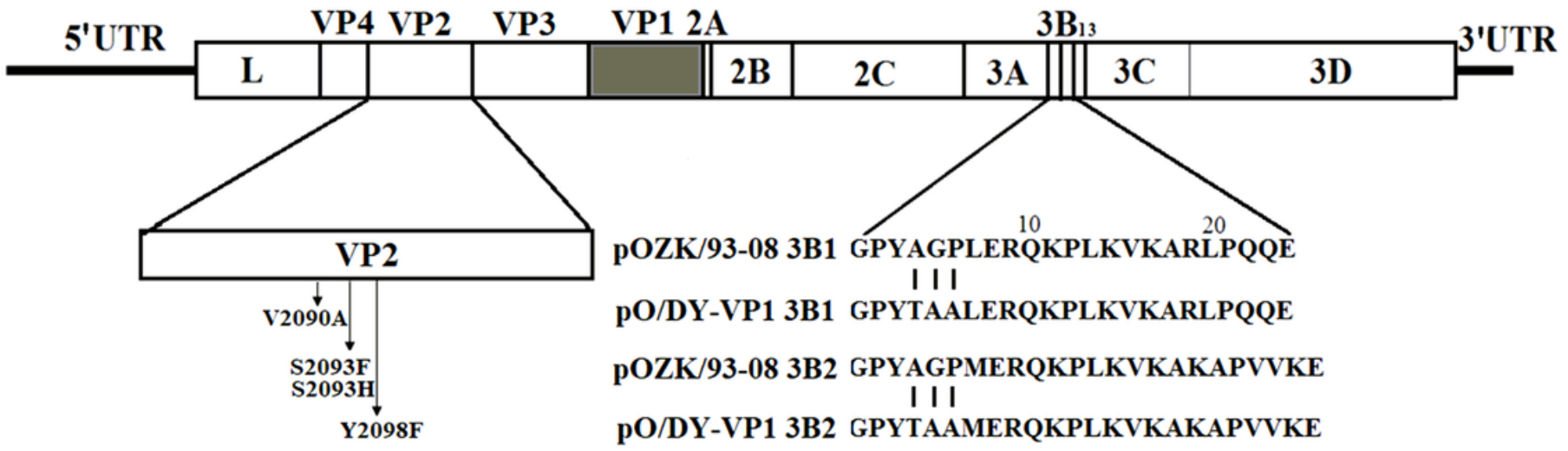

Anti-3A
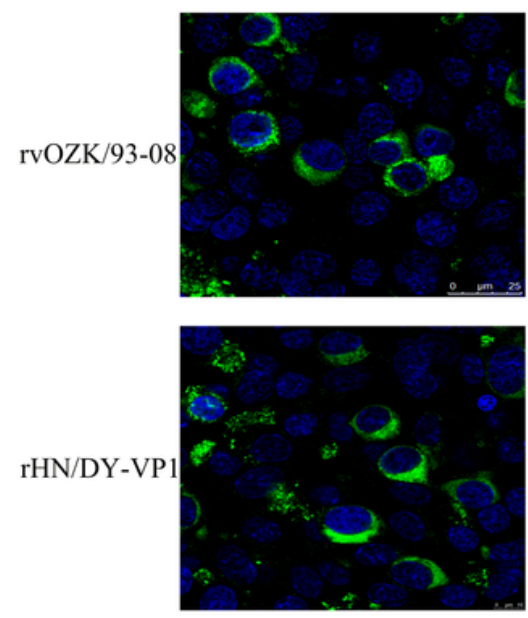

Y2098F

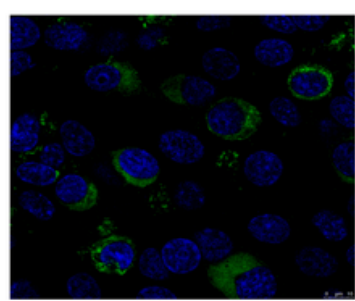

Anti 3B
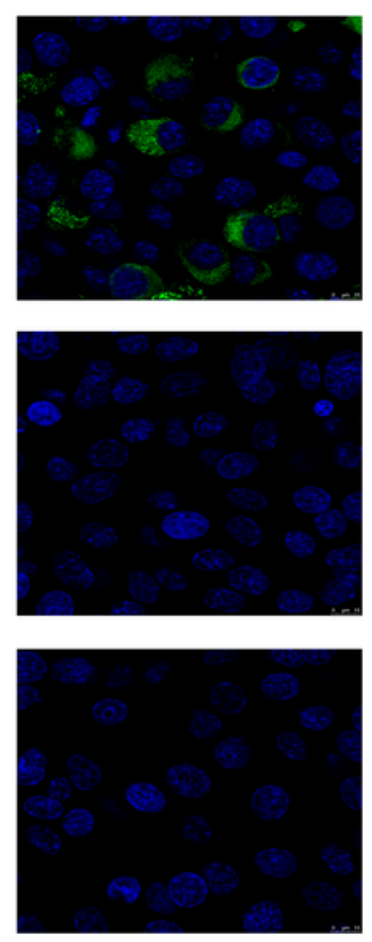

Anti-3A
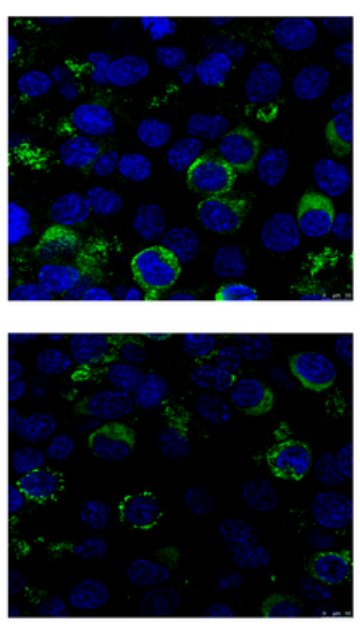

V2090A-S2093F-Y2098F

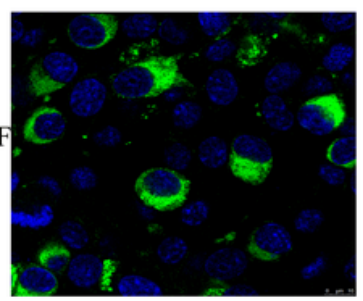

Anti 3B
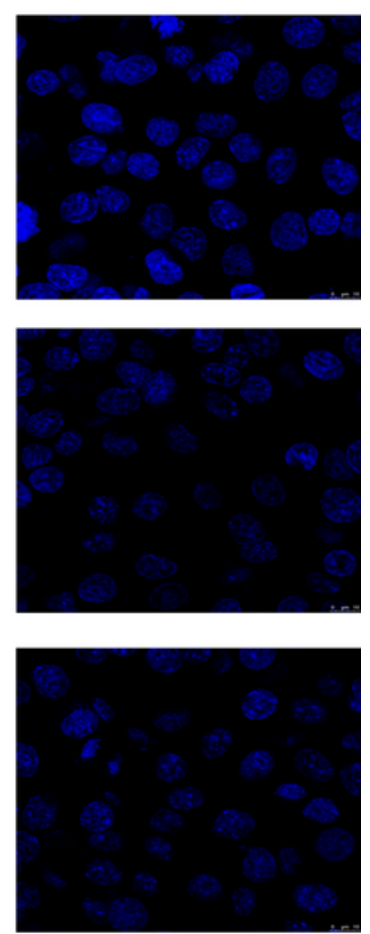

\section{Figure 1}

The design of mutation and the generation of the recombinant FMDV serotype 0 Strains. (a) The structure diagram of the recombinant plasmid pO/DY-VP1 and the thermally stable mutations introduced into pO/DY-VP1. The amino acid residues are numbered respectively for every protein. In the mutant names, the first digit indicates the protein (VP1, VP2, or VP3) and the last three digits the amino acid position; the first letter stand for the original amino acid; the last letter stand for the substituting amino acid. UTR, untranslated region. The 3B1 and 3B2 of the plasmid pO/DY-VP1were mutated (4AGP6- 
4TAA6). (b) The indirect immunofluorescence results of rescued FMDV serotype 0 strains were roughly identical to that of the parental FMDV serotype $O$ strain. Both the parental strain and the recombinant strains could react strongly with MAb 3A24; while all the viral strains completely lack the reaction to MAb 3B4B1. The results of rvOZK/93-08 (recovered from pOZK/93-08) acts as a positive control for the anti3B MAb. rHN/DY-VP1 (the parental strain); Y2098F (rHN/DY-VP1Y2098F, containing Y2098F mutations); V2090A-S2093F (rHN/DY-VP1V2090A-S2093F, containing V2090A-S2093F mutations); V2090A-S2093H (rHN/DY-VP1V2090A-S2093H, containing V2090A-S2093H mutations); V2090A-S2093H-Y2098F (rHN/DY-VP1V2090A-S2093H-Y2098F, containing V2090A-S2093H-Y2098F mutations)

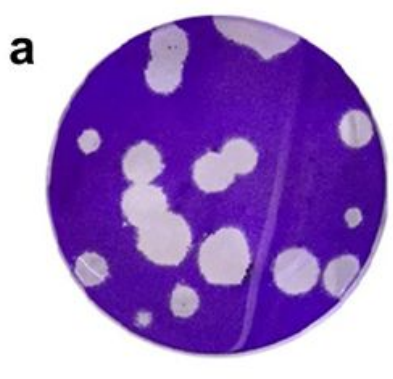

rHN/DY-VP1

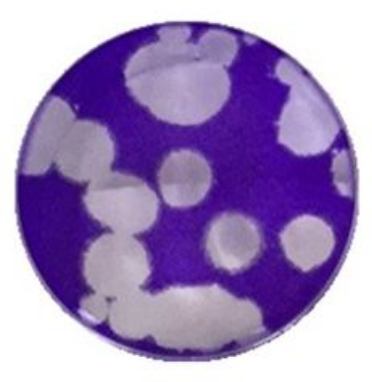

Y2098F

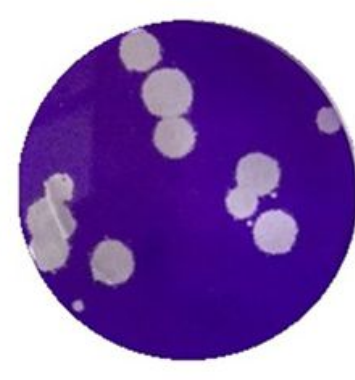

V2090A-S2093F

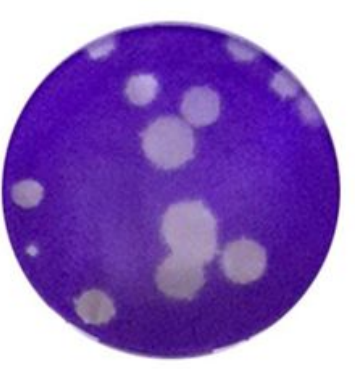

V2090A-S2093H

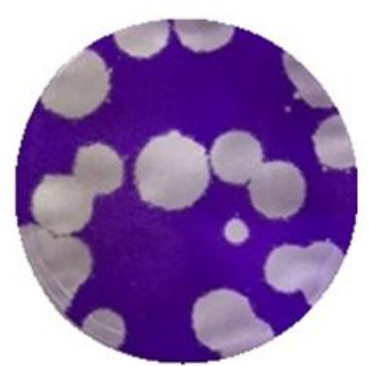

V2090A-S2093H-Y2098F

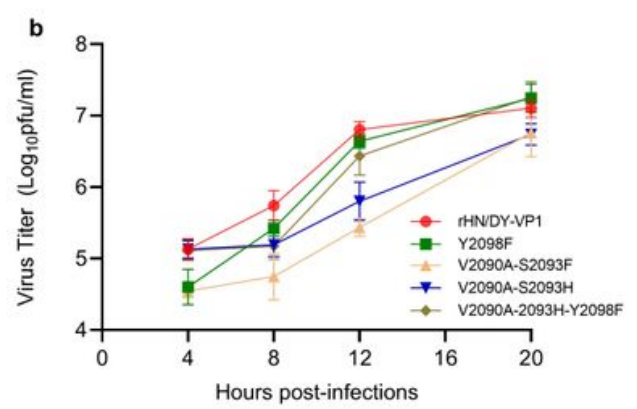

\section{Figure 2}

The growth characteristics of the engineered mutated viral strains. (a) The plaque morphologies of mutated viral strains, including (rHN/DY-VP1Y2098F, rHN/DY-VP1V2090A-S203F, rHN/DY-VP1V2090AS203H and rHN/DY-VP1V2090A-S2093H-Y2098F), formed in BHK-21 cells, were similar to that of the parental virus (rHN/DY-VP1) in BHK-21 cells. (b) The growth kinetics study performed on BHK-21 cells. The average $\log 10$ titers are shown at the different time points $(0,4,8,12$, and $20 \mathrm{~h}$ post absorption), as indicated on the graph, over a 20-h period after the infection with the parental and mutated FMDV serotype 0 strains. Error bars indicate s.d. 
a

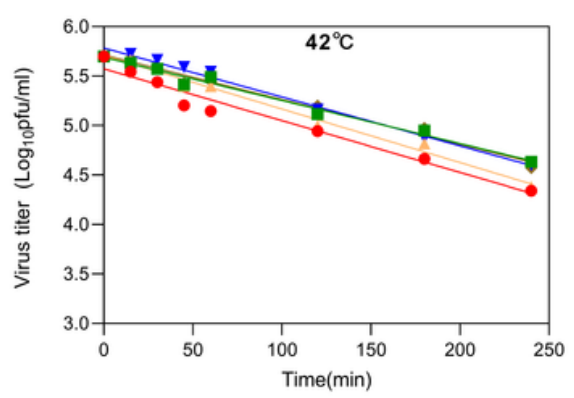

b

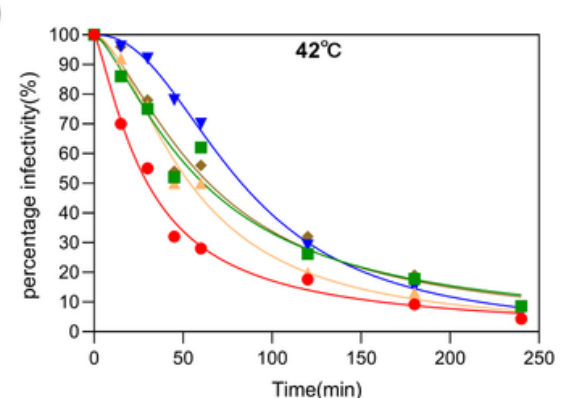

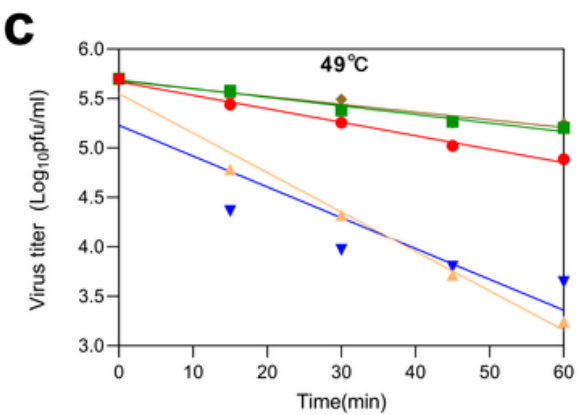

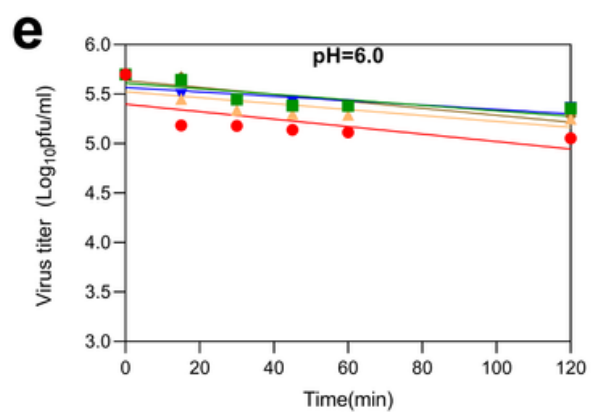

d
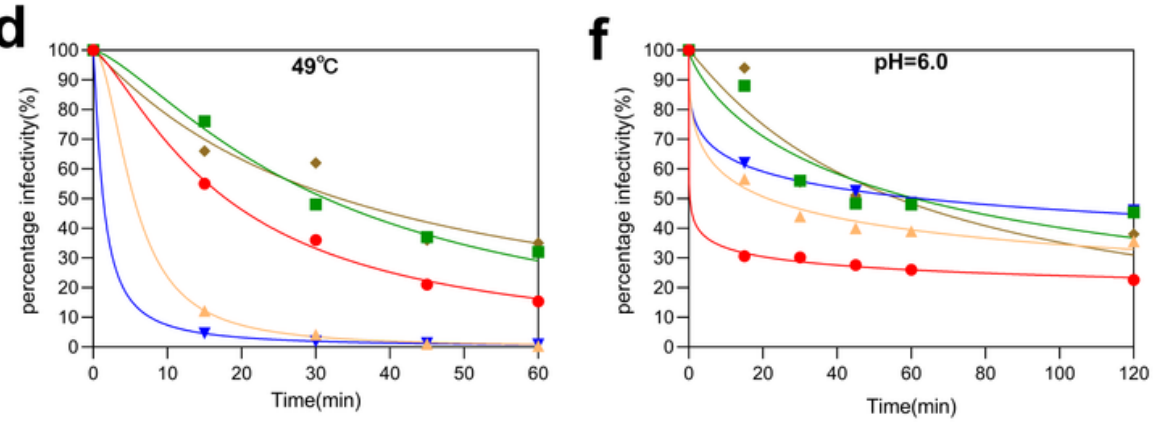

$\rightarrow$ rHN/DY-VP1 $\rightarrow-$ Y2098F

V2090A-S2093F $\rightarrow-\quad$ V2090A-S2093H

V2090A-S2093H-Y2098F

\section{Figure 3}

The thermal and the $\mathrm{pH}$ inactivation kinetics of the FMDV serotype $\mathrm{O}$ parental and the mutated strains tested in duplicate. The inactivation rate of the parental and recombinant viral strains was calculated and plotted following the heat treatment at $42^{\circ} \mathrm{C}$ for $4 \mathrm{~h}(\mathrm{a})$, or at $49^{\circ} \mathrm{C}$ for $1 \mathrm{~h}(\mathrm{c})$, or with the TNE buffer at pH 6.0 for $2 \mathrm{~h}(\mathrm{e})$. The average $\log 10$ titers of the viral strains assayed with two different inactivation experiments are shown. The respective logarithmic values of the titers of the viral strains at the different time points $(0,15,30,45,60,90,120,180$, and 240 min post the infection) were linearly fitted and the slopes were assayed, the average titers of the viral strains following heat treatment at $42^{\circ} \mathrm{C}(\mathrm{b})$ or $49^{\circ} \mathrm{C}(\mathrm{d})$ or following the $\mathrm{pH}$ treatment (f) at the aforementioned time points were compared with the initial titers to calculate the remaining percentages of the infectious viral virions left over times 
a
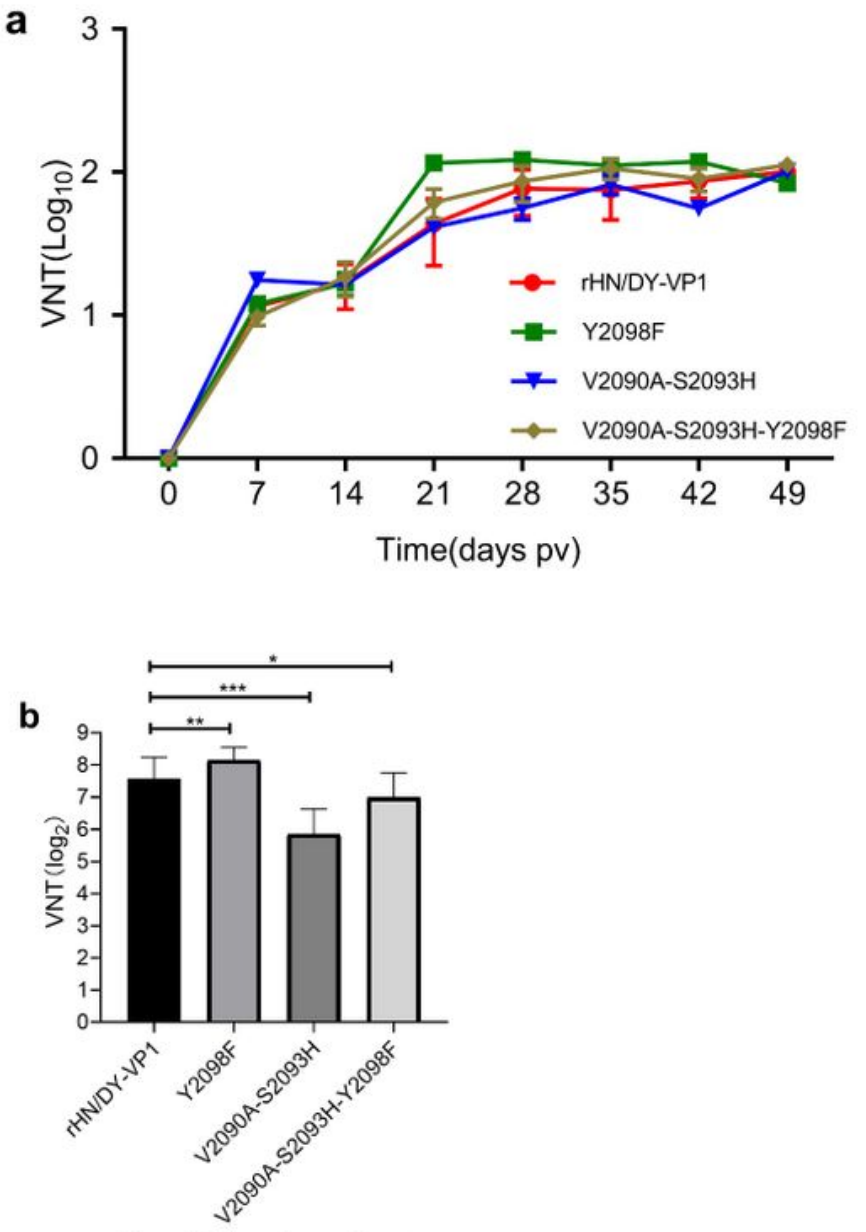

4-month long-term storage

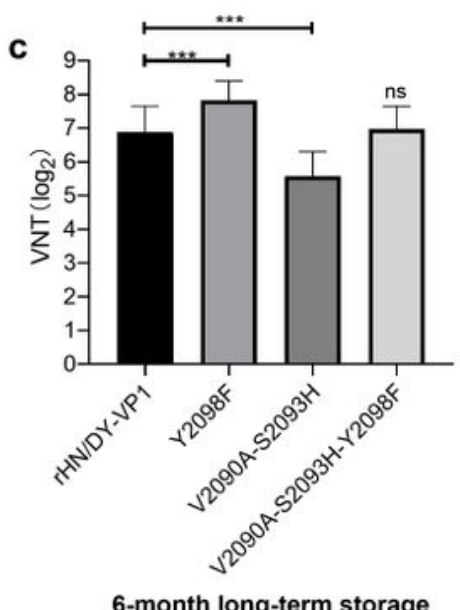

\section{Figure 4}

The Immunogenicity of the inactivated wild-type and the thermally stabilized viral strains. (a) The Mean viral neutralizing-antibody titer (VNT) $(\log 10))$ with the pigs vaccinated with the purified inactivated parental and mutant viral strains. Groups of pigs, with four pigs in each group were vaccinated with either the parental viral strain rHN/DY-VP1 or the mutated viral strains rHN/DY-VP1Y2098F(Y2098F), rHN/DYVP1V2090A-S2093H (V2090A-S2093H), and rHN/DY-VP1V2090A-S2093H-Y2098F (V2090A-S2093H- 
Y2098F). The blood then were sampled and assayed at 0, 7, 14, 21, 28, 35 and 42 days pv. Error bars indicate s.d. (b) The Virus Neutralization Test (VNT)s of guinea pigs were carried out to assess the immunogenic effectiveness of the inactivated virions after 4-month of long-term storage. Before the immunization, the formulated vaccines were stored for 4 months at $4{ }^{\circ} \mathrm{C}$. Two groups of guinea pigs, with ten in each group were vaccinated with either the parental strain or the mutated strains, and VNTs were then assessed at 28 days pv. Error bars indicate s.d. ${ }^{*} P<0.05$; ${ }^{*} P<0.01, * \star * P<0.001$. (c) The VNTs with the guinea pigs were used to assess the immunogenicity of the inactivated virions after a 6-month of long-term storage. The aliquots of the putative vaccines formulated with the parental and mutated viral strains were almost equal to each other after the putative vaccines stored for 6 months at $4{ }^{\circ} \mathrm{C}$ before the

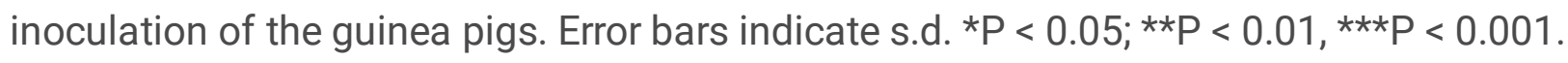

